\title{
Phase Separation and Microwave Response of Epitaxial and Polycrystalline Manganite Films
}

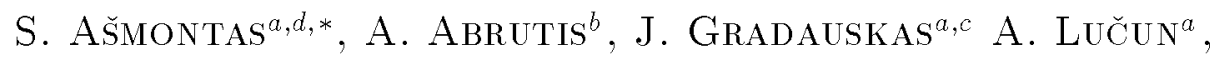 \\ A. Oginskis ${ }^{a}$, V. Plaušinaitien $\dot{E}^{a, b}$, A. SužIedélis ${ }^{a, c}$ \\ AND B. VENGALIS ${ }^{a, c}$ \\ ${ }^{a}$ Semiconductor Physics Institute \\ A. Goštauto 11, 2600 Vilnius, Lithuania \\ ${ }^{b}$ Department of General and Inorganic Chemistry, Vilnius University \\ Naugarduko 24, 2006 Vilnius, Lithuania \\ ${ }^{c}$ Vilnius Gediminas Technical University \\ Sauletekio al. 11, 2040 Vilnius, Lithuania \\ ${ }^{d}$ KTU Panevėžys Institute, Klaipèdos 3, 5319 Panevėžys, Lithuania
}

\begin{abstract}
The resistance, magnetoresistance, and resistance response under microwave irradiation ( $f=10$ and $35 \mathrm{GHz}$ ) were measured for epitaxial and polycrystalline $\mathrm{La}_{0.67} \mathrm{Ca}_{0.33} \mathrm{MnO}_{3}$ and $\mathrm{La}_{0.67} \mathrm{Sr}_{0.33} \mathrm{MnO}_{3}$ thin films in the temperature range $78 \div 300 \mathrm{~K}$. The microwave induced resistance increase observed for the epitaxial films in a narrow temperature range below the ferromagnetic to paramagnetic transition temperature $T_{c}$ certifies coexistence of low resistance (ferromagnetic) and high resistance (paramagnetic) regions in the manganites. Resistance of polycrystalline films decreased under microwave irradiation in a wide temperature range below $T_{c}$. The effect was explained in terms of microwave assisted hopping of carriers in high resistance regions formed at grain boundaries of the polycrystalline films.
\end{abstract}

PACS numbers: 75.47.Gk, 75.47.Lx, 07.57.Kp

\section{Introduction}

Recent increased interest in rare-earth manganese perovskites [1] is conditioned by promising application possibilities of their "colossal" magnetoresistance

* corresponding author; e-mail: as@uj.pfi.lt 
(CMR) $[2,3]$. Considerable advance in the understanding of the origin of the CMR was achieved after theoretical predictions of the intrinsically inhomogeneous character of the ground state in perovskites resulting in a strong tendency towards phase separation [4]. A number of experimental works [5] evidently supported the idea of phase separation. Recently, we demonstrated that microwave (MW) irradiation of $\mathrm{La}_{0.67} \mathrm{Ca}_{0.33} \mathrm{MnO}_{3}$ films can be used to prove the coexistence of metallic ferromagnetic (FM) and high resistance paramagnetic (PM) phases [6, 7].

Both epitaxial and polycrystalline $\mathrm{La}_{0.67} \mathrm{Ca}_{0.33} \mathrm{MnO}_{3}$ (LCMO) as well as $\mathrm{La}_{0.67} \mathrm{Sr}_{0.33} \mathrm{MnO}_{3}$ (LSMO) thin films were investigated in this work. The choice of different manganite materials characterized by different crystalline quality, different electrical and magnetic properties, as well as the use of two different microwave frequencies enabled us to generalize our previous experimental findings concerning phase separation in epitaxial manganite films and to elucidate the importance of intergrain boundaries on high frequency transport in polycrystalline materials.

\section{Experimental procedure}

LCMO thin films with typical thickness of 150-200 nm were magnetron sputtered at $750^{\circ} \mathrm{C}$ under $\mathrm{Ar}: \mathrm{O}_{2}(1: 1)$ gas pressure of about $30 \mathrm{~Pa}$ onto lattice-matched $\mathrm{NdGaO}_{3}(100)$ and cubic $\mathrm{MgO}(100)$ exhibiting a significant lattice mismatch of about $9 \%$. Thin LSMO films were grown also on $\mathrm{NdGaO}_{3}(100)$ and cubic $\mathrm{MgO}(100)$ substrates using a vertical hot wall injection chemical vapour deposition (CVD) reactor [8]. The deposition was performed at $825^{\circ} \mathrm{C}$ in $\mathrm{Ar}: \mathrm{O}_{2}(2: 1)$ gas mixture atmosphere. Typical dimensions of the substrates were $10 \times 4 \mathrm{~mm}^{2}$. Both $\Theta-2 \Theta \mathrm{X}$-ray diffraction measurements and reflected high-energy electron diffraction revealed the epitaxial quality of LCMO and LSMO films grown on $\mathrm{NdGaO}_{3}$, while both the LCMO and LSMO films grown on $\mathrm{MgO}(100)$ were polycrystalline, i.e. they showed preferential (100) orientation with certain negligible amount of [111]-axis oriented grains. Silver coatings of $1 \mathrm{~mm}$ width were evaporated onto the films to perform low frequency electrical and microwave measurements. The space between metallic contacts was made wider $(\approx 7 \mathrm{~mm})$ than the height of the lowered waveguide $(\approx 4 \mathrm{~mm})$ with the aim to minimize microwave detection in the contacts.

Low frequency electrical transport measurements were conducted between $78 \mathrm{~K}$ and $300 \mathrm{~K}$ using a two-probe ac technique. A driving ac current of $1 \mu \mathrm{A}$ and a frequency of $131 \mathrm{~Hz}$ were used. The negative magnetoresistance $\mathrm{MR}=$ $\left(R_{0}-R_{\mathrm{H}}\right) / R_{0}$ was determined from two separate resistance measurements, where $R_{0}$ and $R_{\mathrm{H}}$ are resistance values without and with external magnetic field, respectively.

For the microwave measurements, the samples were placed into the centre of a lowered rectangular waveguide perpendicular to the flat waveguide walls leaving the contact pads outside the waveguide. The electrical component of the microwave field was parallel to the film surface. The microwave electric field with a frequency 
of $10 \mathrm{GHz}$ and $35 \mathrm{GHz}$, pulse duration of $2 \mu \mathrm{s}$, repetition rate of $40 \mathrm{~Hz}$ and a maximal power of $8 \mathrm{~kW}$ and $800 \mathrm{~W}$, respectively, was applied. The samples under investigation were biased with dc current of $40 \mu \mathrm{A}$ using in series connected $110 \mathrm{k} \Omega$ resistor. The dynamics of the dc voltage drop across the sample following the dynamics of the dc resistance was measured using oscilloscope and CCD camera.

\section{Results and discussion}

Figure 1 demonstrates typical resistance versus temperature for the manganite films of various crystalline quality. Both epitaxial and polycrystalline films showed a resistance increase with cooling above the PM-FM transition temperature $T_{\mathrm{c}}$ followed by resistance drop at $T<T_{\mathrm{c}}$. The polycrystalline $\mathrm{LCMO} / \mathrm{MgO}$ and LSMO/MgO films exhibited the PM-FM phase transition at lower temperatures and in a wider temperature range compared to the corresponding epitaxial films.

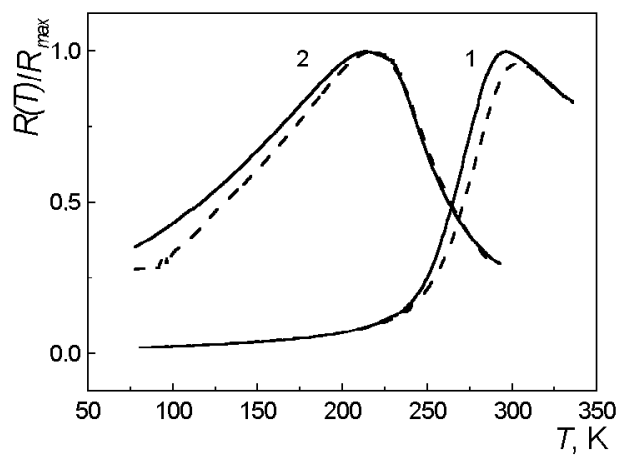

Fig. 1. Resistance versus temperature measured for the epitaxial $\mathrm{La}_{0.67} \mathrm{Sr}_{0.33} \mathrm{MnO}_{3} / \mathrm{NdGaO}_{3}$ (1) and polycrystalline $\mathrm{La}_{0.67} \mathrm{Ca}_{0.33} \mathrm{MnO}_{3} / \mathrm{MgO}$ (2) thin films at $B=0 \mathrm{~T}$ (solid line) and $B=0.8 \mathrm{~T}$ (dashes).

For all the films investigated, the application of magnetic field resulted in a lowering of their resistance peaks and slight shift of the corresponding $T_{\mathrm{c}}$ values to higher temperatures. For the epitaxial films, high magnetoresistance values were measured in a relatively narrow temperature region below $T_{\mathrm{c}}$ while for the polycrystalline films the highest MR values were indicated at the lowest temperatures.

In Fig. 2 we show the MW response (MWR), i.e. the microwave pulse induced dc resistance change for the epitaxial LSMO film at $f=10 \mathrm{GHz}$ and $f=35 \mathrm{GHz}$ frequencies. The peculiarities shown in this figure are in a good accordance to similar results reported by us earlier for high quality LCMO films [6, 7]. Following Fig. 2 we point out significant increase in MWR with MW frequency. Summarizing our results for the epitaxial films we point out: (1) slight resistance decrease in the epitaxial manganite films under MW power in the PM-phase region, (2) polarity 


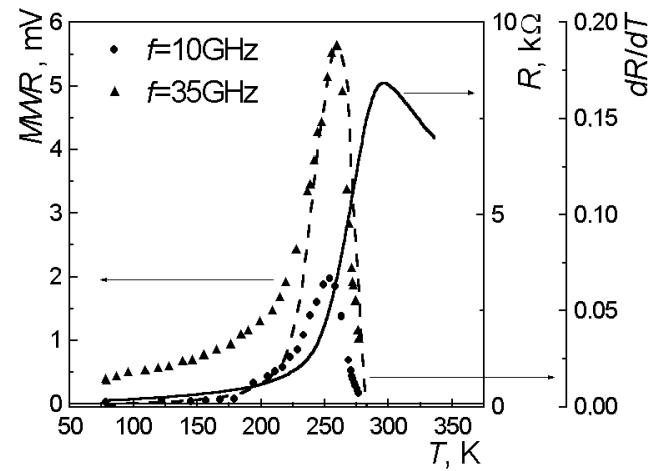

Fig. 2. Temperature dependence of MW pulse induced dc resistance response, MWR, measured for the epitaxial $\mathrm{La}_{0.67} \mathrm{Sr}_{0.33} \mathrm{MnO}_{3} / \mathrm{NdGaO}_{3}$ film at $f=10 \mathrm{GHz}(\bullet)$ and $f=35 \mathrm{GHz}$ (full $\triangle$ ). The temperature derivative (dashed line) of the film resistance (solid line) is depicted for a comparison.

change of the MWR signal with cooling just above the PM-FM transition temperature and (3) the subsequent resistance increase with applied MW pulse in the FM phase region at lower temperatures.

Figure 3 depicts the MWR measured for the polycrystalline LCMO/MgO film at $f=10 \mathrm{GHz}$ frequency. In contrast to the epitaxial films, a decrease in the electrical resistance under influence of a microwave pulse was seen for the polycrystalline films in the whole temperature range both above and below the PM-FM transition temperature.

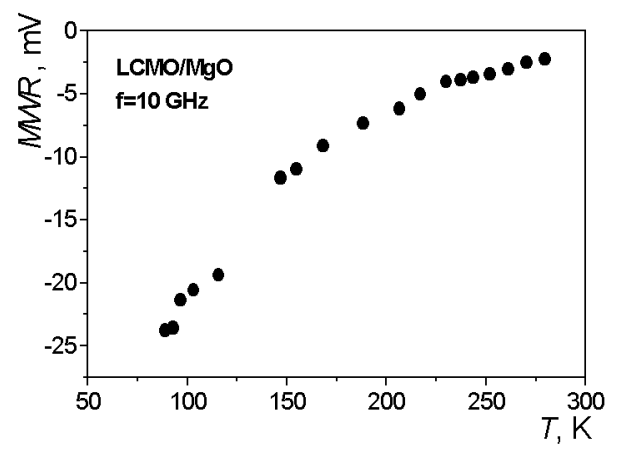

Fig. 3. Typical temperature dependence of the $\mathrm{MW}$ induced resistance response of the polycrystalline $\mathrm{La}_{0.67} \mathrm{Ca}_{0.33} \mathrm{MnO}_{3} / \mathrm{MgO}$ film at $f=10 \mathrm{GHz}$.

At room temperature the absolute value of resistance response of the epitaxial films on MW radiation was linear up to the maximal radiation power of $8 \mathrm{~kW}$ while at $T=80 \mathrm{~K}$ this linearity was only indicated up to $\approx 1 \mathrm{~kW}$ (see curves 1,2 in Fig. 4). It is worth noting that nonlinear properties of MWR (curves 4, 5) were also indicated for the polycrystalline films at low temperatures. 


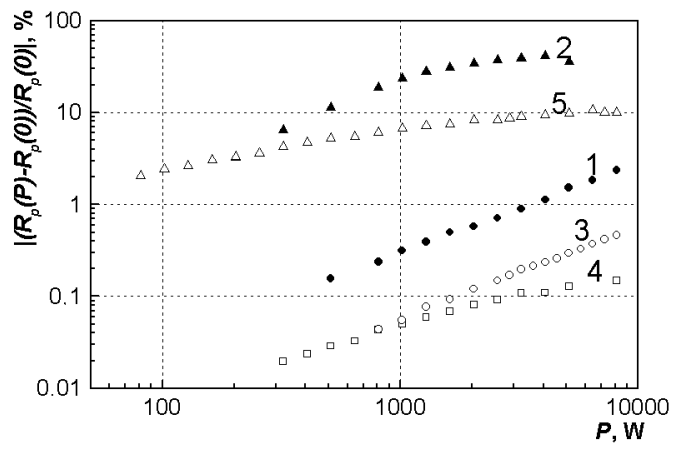

Fig. 4. The dependence of relative resistance change of epitaxial $\mathrm{La}_{0.67} \mathrm{Sr}_{0.33} \mathrm{MnO}_{3}$ $(1,2)$ and polycrystalline $\mathrm{La}_{0.67} \mathrm{Ca}_{0.33} \mathrm{MnO}_{3}(3,4,5)$ films on microwave power at $T=300 \mathrm{~K}(1,3), 130 \mathrm{~K}(4)$, and $80 \mathrm{~K}(2,5)$.

Relaxation character of the electrical resistance of the epitaxial manganite film was different above and below the phase transition temperature, as can be seen from Fig. 5. In the PM phase the resistance decrease can be properly fitted using a single exponential law. Below the transition temperature, two exponents are needed for fitting: a fast equilibration process (of about $0.8 \mu \mathrm{s}$ ) between the semiconductor like PM and metallic FM phases takes place at first, and then the slower relaxation (in the tens of microseconds range) of the resistance change occurs due to temperature equilibration between the film and the substrate.

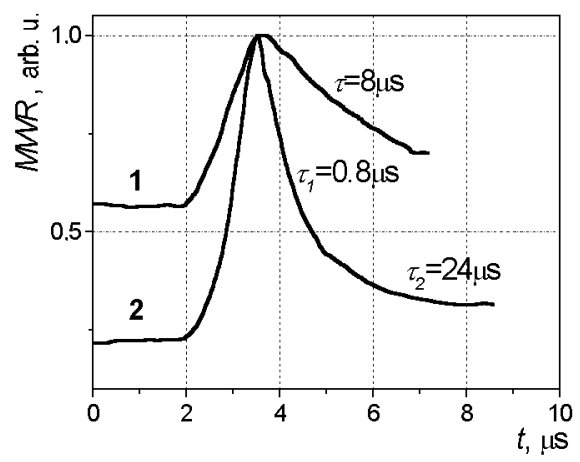

Fig. 5. Relaxation of the microwave induced dc resistance change of epitaxial $\mathrm{La}_{0.67} \mathrm{Sr}_{0.33} \mathrm{MnO}_{3}$ film measured above and below the Curie temperature $T_{\mathrm{c}} . T=265 \mathrm{~K}$ (1), $259 \mathrm{~K}(2)$.

High magnetoresistance values measured for the highest crystalline quality manganite films just below their PM-FM transition temperature are due to a shift of their $T_{c}$ values caused by the applied magnetic field (CMR effect) [2, 3]. Relatively high magnetoresistance values measured for the polycrystalline LSMO/MgO and LCMO/MgO films at low temperatures may be understood assuming tunnelling magnetoresistance effect due to the existing grain boundaries. 
To elucidate the origin of microwave induced resistance change, i.e. the microwave response for epitaxial and polycrystalline manganite films (see Figs. 2 and 3) first of all it is worth to consider possible sample heating by the absorbed MW power (bolometric effect). Due to this heating, the resultant MWR for the films should be proportional to the temperature derivative of their resistance, TCD $=\mathrm{d} R / \mathrm{d} T$ (see the corresponding TCD versus $T$ curve displayed in Fig. 2). Following Fig. 2, one can conclude that the bolometric effect has major influence on MWR for the epitaxial films at $T>T_{\mathrm{c}}$ as well as just below $T_{\mathrm{c}}$ where the TCD reaches maximal negative value. Certainly, both the polarity and temperature dependence of the MWR signal in this temperature region are in a good accordance with those of TCD. However, it can be seen from the same figure that at lower temperatures the MWR for the film is much higher than that expected from the bolometric effect. We associate the origin of this enhanced microwave response of epitaxial manganite films below their PM-FM phase transition temperature $T_{\mathrm{c}}$ with the dynamical coexistence of semiconducting and metallic phases. When the material is homogeneous (either semiconducting or metallic), the microwave electric field is uniformly absorbed in the whole sample and its resistance change is proportional to the absorbed MW power. However, in a nonhomogeneous material with coexisting metallic and high resistance regions, the effect of the absorbed MW power on dc resistance change will be different. DC current in this case will flow mainly through low resistance FM regions although the resultant sample resistance will depend mainly on high resistance PM-phase regions separating the metallic FM islands in the transition region. In a nonhomogeneous sample, the MW electric field will be distributed much more uniformly compared to the applied dc field and thus, the MW power will be mainly absorbed in the metallic regions. The resistance change in this case will be more evident than that in a homogeneous sample due to a nonuniform sample heating by MW irradiation resulting in more effective shrinkage of the metallic regions.

Strong nonlinearity of the MWR with the MW power $P$ as well as very fast dynamics of the MWR observed for the epitaxial films at low temperatures (see Fig. 5) may be considered as additional data supporting the proposed phase separation model. On the other hand, at room temperature $\left(T>T_{c}\right)$ the observed linear MWR and relatively slow film resistance dynamics support dominating role of the bolometric effect both for the epitaxial and polycrystalline films.

Finally, a new model based on current flow in presence of high resistance grain boundaries in polycrystalline material needs to be carried out to explain significant MW induced resistance decrease for the $\mathrm{LSMO} / \mathrm{MgO}$ and $\mathrm{LCMO} / \mathrm{MgO}$ films at low temperatures. Our experimental data demonstrate that MW irradiation may have influence on resistance of polycrystalline manganite films via enhanced hopping probability of carriers in high resistance intergrain media. 


\section{Conclusions}

Coexistence of metallic FM- and high resistance PM-phases in the epitaxial LCMO and LSMO films was proved by: (1) enhanced MW-induced resistance response in a narrow temperature region below the Curie temperature, (2) fast resistance relaxation process at $T<T_{c}$, and (3) considerable frequency dependence of the MW response. Determinant factors for high frequency transport in polycrystalline manganite films were attributed to the presence of intergrain boundaries and MW irradiation assisted hopping of carriers in a high resistance intergrain media.

\section{Acknowledgments}

This work was partially supported by the EC within the MULTIMETOX network and CE project PRAMA and Lithuanian Science Foundation Grant T-49. We acknowledge Prof. Jurgis Kundrotas for useful discussions.

\section{References}

[1] G.H. Jonker, J.H. van Santen, Physica 16, 337 (1950).

[2] R. von Helmholt, J. Wecker, B. Holzapfel, L. Schultz, K. Samwer, Phys. Rev. Lett. 71,2331 (1993).

[3] S. Jin, T.H. Tiefel, M. McCormak, R.A. Fastnacht, R. Ramesh, L.H. Chen, Science 264, 413 (1994).

[4] A. Moreo, S. Yunoki, E. Dagotto, Science 283, 2034 (1999).

[5] R.W. Li, Z.R. Zhang, Q.A. Li, J.R. Sun, G.J. Wang, Z.H. Cheng, Z.H. Wang, S.Y. Zhang, B.S. Han, B.G. Shen, J. Appl. Phys. 92, 7404 (2002).

[6] A. Lisauskas, A. Sužiedèlis, A. Lučun, A. Oginskis, J. Gradauskas, B. Vengalis, S. Ašmontas, Lith. J. Phys. 42, 331 (2002).

[7] A. Lisauskas, A. Sužiedèlis, A. Lučun, A. Oginskis, J. Gradauskas, B. Vengalis, S. Ašmontas, Solid State Phenom. 94, 257 (2003).

[8] A. Abrutis, V. Kubilius, V. Bigelytè, A. Teišerskis, Z. Šaltytè, J.P. Senateur, F. Weiss, Mater. Lett. 31, 201 (1997). 